\title{
Function Synthesis of the Planar 5R Mechanism Using Least Squares Approximation
}

\author{
Gökhan Kiper, Barış Bağdadioğlu and Tunç Bilgincan
}

\begin{abstract}
In this paper, the problem of function generation synthesis of planar 5R mechanism is studied using the least squares approximation method with equal spacing of the design points. The study represents a case study for analytical function generation of multi-degrees-of-freedom systems. The planar 5R mechanism is designed with a fixed input joint and a moving input joint adjacent to the first input, whereas the remaining fixed joint is the output joint. The input/output relationship of the mechanism is expreseed as an objective function in polynomial form with four unknown construction parameters. . The objective function involves nonlinearities, however the problem is linearized using Lagrange variables. The linear system is solved and finally the construction parameters of the mechanism are determined. A numerical example is presented as a case study.
\end{abstract}

Key words: function generation, planar 5R mechanism, least squares approximation, equal spacing

\section{Introduction}

One of the research areas in Rasim Alizade Mechatronics Laboratory in IzTech addresses analytical kinematic synthesis of multi degrees-of-freedom (dof) systems. Although analytical kinematic synthesis methods for single-dof mechanisms are widely studied $[1,2]$, usually numerical optimization methods are utilized for dimensioning multi-dof mechanisms (ex. see [3]). There are a few studies on analytical synthesis methods for multi-dof systems [4,5]. Recently we worked on function

Gökhan Kiper, Barış Bağdadioğlu and Tunç Bilgincan

İzmir Institue of Technology, 35430, İzmir, Turkey e-mail: gokhankiper@iyte.edu.tr, barisbagdadiogludiyte.edu.tr, tuncbilgincan@iyte.edu.tr 
synthesis of a planar 5R mechanism [6] and a spherical 5R mechanism [7]. The present study differs from [6] in selection of one of the inputs, and also a different synthesis method is utilized.

Theree widely used approximation methods for kinematic synthesis are the interpolation, least squares and Chebyshev approximation methods [8]. In this study we employ the least squares method $[8,10]$. In any of these methods, first an objective function is defined using the input/output $(\mathrm{I} / \mathrm{O})$ relationship of the mechanism and then an approximation in polynomial form is sought for the objective function. The approximation is performed for a certain number of design points on the domain of the inputs. For the interpolation approximation, these design points are precision points at which the mechanism exactly generates the required function value, while for the least squares and Chebyshev approximations the aim is to minimize the nonzero errors at the design poitns. The approximation error depends on the selection of design points and optimizing the selection is a big issue [11]. In case of single-dof mechanisms the domain for the design points is just a line segment, while in general for an $\mathrm{n}$-dof mechanism the domain will be an $\mathrm{n}$-dimensional compact space. Specifically, for a 2-dof mechanism the domain is typically rectangular.

In $[6,7]$ we emolyed Chebyshev approximation. In [6] we had five design points, four of which we located on the sides of the rectangular domain. In [7] we tried a different type of spacing for the design points, which we called regional spacing. In regional spacing the design points are located in distinct regions in the domain and since the Chebyshev approximation method involves iterations, the design points relocated in each iteration step, but they are forced to remain in their respective regions. In least squares approximation there is no iteration. As the name implies, the aim is to minimize the sum of the squares of the errors at the design points. In this study we make use of equal spacing for the design points. For single-dof mechanisms generally Chebyshev spacing gives superior results $[8,12]$, however the comparison of the methods for 2-dof mechanisms is yet to be done. Although we obtained quite good results with Chebyshev approximation [6,7], we expect that least squares approximation may yield better results at least for some functions and some mechanisms.

\section{Formulation}

In this study, the input variables $\theta$ and $\phi$ of the planar 5R mechanism are associated with one of the fixed joints and the adjacent floating joint. The output variable $\psi$ is associated with the remaining fixed joint. In practice, an extra parallelogram loop can be employed in order to actuate the mechanism at fixed joints (Figure 1). Since the scale of the mechanism does not affect the I/O relationship, without loss of generality we may assume that the fixed link length is 1 . The construction parameters are $a, b, d$ and e shown in Figure 1. 


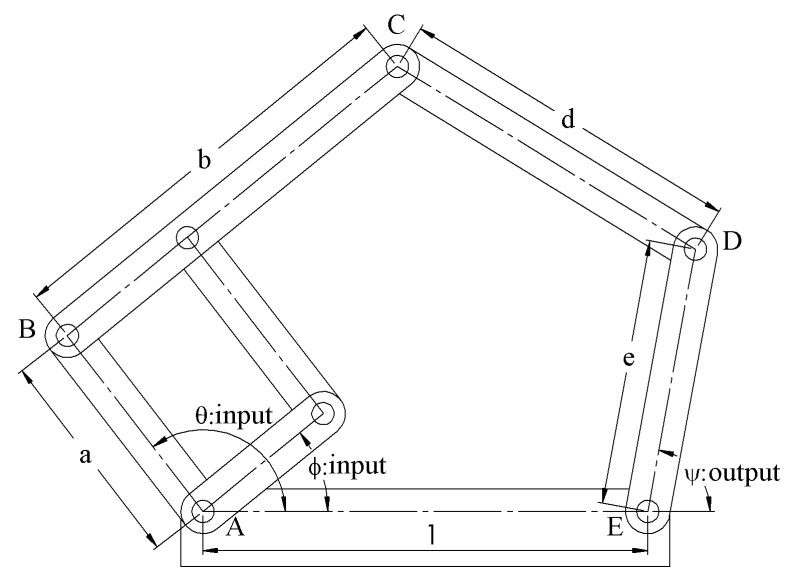

Fig. 1 The construction parameters and joint variables of the 5R mechanism

The I/O relationship for the mechanism is obtained as follows:

$$
\begin{gathered}
|\overrightarrow{C D}|=|\overrightarrow{A E}+\overrightarrow{E D}-\overrightarrow{A B}-\overrightarrow{B C}| \\
\Rightarrow(a C \theta+b C \phi-1-e C \psi)^{2}+(a S \theta+b S \phi-e S \psi)^{2}=d^{2}
\end{gathered}
$$

where $C$ and $S$ represent the cosine and sine functions, respectively. Rearranging Eq. (1) in polynomial form:

$$
\sum_{j=1}^{6} P_{j} f_{j}(\mathbf{x})-F(\mathbf{x})=0
$$

where $\mathbf{x}=\{\theta, \phi, \psi\}$, whereas $\left\{P_{j}\right\}_{1}^{6},\left\{f_{j}(\mathbf{x})\right\}_{1}^{6}$ and $F(\mathbf{x})$ are defined as:

$$
\begin{gathered}
P_{1}=\frac{-1-a^{2}-b^{2}+d^{2}-e^{2}}{2 e}, P_{2}=a, P_{3}=b, P_{4}=\frac{a}{e}, \\
P_{5}=P_{3} P_{4}=\lambda_{1}=\frac{a b}{e}, P_{6}=\frac{P_{5}}{P_{2}}=\lambda_{2}=\frac{b}{e}, \\
f_{1}(\mathbf{x})=1, f_{2}(\mathbf{x})=\cos (\theta-\psi), f_{3}(\mathbf{x})=\cos (\phi-\psi), f_{4}(\mathbf{x})=\cos \theta, \\
f_{5}(\mathbf{x})=-\cos (\theta-\phi), f_{6}(\mathbf{x})=\cos \phi \text { and } F(\mathbf{x})=\cos \psi
\end{gathered}
$$

There are four construction parameters, but six $P_{j}$ 's. Therefore $P_{5}$ and $P_{6}$ are defined in terms of the other $P_{j}$ 's and two Lagrange parameters $P_{5}=\lambda_{1}$ and $P_{6}=\lambda_{2}$ are introduced as two more construction parameters. In order to linearize the system, let $P_{j}=\ell_{j}+m_{j} \lambda_{1}+n_{j} \lambda_{2}$ for $j=1,2,3,4$. Eq. (2) becomes

$$
\sum_{j=1}^{4}\left(\ell_{j}+m_{j} \lambda_{1}+n_{j} \lambda_{2}\right) f_{j}(\mathbf{x})+\lambda_{1} f_{5}(\mathbf{x})+\lambda_{2} f_{6}(\mathbf{x})-F(\mathbf{x})=0
$$


Eq. (4) should be satisfied for all $\mathbf{x}$, hence the coefficients of $\lambda_{1}, \lambda_{2}$ and the rest can be dissected as follows:

$$
\begin{aligned}
& \sum_{j=1}^{4} \ell_{j} f_{j}(\mathbf{x})-F(\mathbf{x})=0 \\
& \sum_{j=1}^{4} m_{j} f_{j}(\mathbf{x})+f_{5}(\mathbf{x})=0 \\
& \sum_{j=1}^{4} n_{j} f_{j}(\mathbf{x})+f_{6}(\mathbf{x})=0
\end{aligned}
$$

In least squares approximation the number of design points, $\mathrm{N}$, is necessarily greater than the number of construction parameters and we aim to minimize the sum of the squares of the errors at these design points. At each design point $\mathbf{x}_{i}$ for $i=1, \ldots, N$, the sum of the squares of the errors corresponding to Eqs. (5-7) are defined as

$$
\begin{gathered}
S_{\ell}=\sum_{i=1}^{N}\left[\sum_{j=1}^{4} \ell_{j} f_{j i}-F_{i}\right]^{2} \\
S_{m}=\sum_{i=1}^{N}\left[\sum_{j=1}^{4} m_{j} f_{j i}+f_{5 i}\right]^{2} \\
S_{n}=\sum_{i=1}^{N}\left[\sum_{j=1}^{4} n_{j} f_{j i}+f_{6 i}\right]^{2}
\end{gathered}
$$

where $f_{j i}=f_{j}\left(\mathbf{x}_{i}\right), f_{5 i}=f_{5}\left(\mathbf{x}_{i}\right), f_{6 i}=f_{6}\left(\mathbf{x}_{i}\right)$ and $F_{i}=F\left(\mathbf{x}_{i}\right)$. In order to find the minimum of the sum of the squares, we set the derivatives of Eqs. (8-10) with respect to $\ell_{j}, m_{j}, n_{j}$ to zero for $j=1,2,3,4$ to obtain

$$
\begin{gathered}
\frac{1}{2} \frac{\partial S_{\ell}}{\partial \ell_{j}}=\sum_{i=1}^{N}\left[f_{1 i} \ell_{1}+f_{2 i} \ell_{2}+f_{3 i} \ell_{3}+f_{4 i} \ell_{4}-F_{i}\right] f_{j i}=0 \\
\frac{1}{2} \frac{\partial S_{m}}{\partial m_{j}}=\sum_{i=1}^{N}\left[f_{1 i} m_{1}+f_{2 i} m_{2}+f_{3 i} m_{3}+f_{4 i} m_{4}+f_{5 i}\right] f_{j i}=0 \\
\frac{1}{2} \frac{\partial S_{n}}{\partial n_{j}}=\sum_{i=1}^{N}\left[f_{1 i} n_{1}+f_{2 i} n_{2}+f_{3 i} n_{3}+f_{4 i} n_{4}+f_{6 i}\right] f_{j i}=0
\end{gathered}
$$

For $j=1,2,3,4$ Eq. (11) constitute a linear set of 4 equations in unknowns $\ell_{1}, \ell_{2}, \ell_{3}, \ell_{4}$ and similarly Eqs. (12) and (13) are respectively linear in $m_{1}, m_{2}, m_{3}, m_{4}$ and $n_{1}, n_{2}, n_{3}, n_{4}$. Writing Eqs. (11-13) in matrix form:

$$
\begin{array}{r}
{\left[A_{j k}\right]\left[\ell_{j}\right]=\left[b_{j}\right]} \\
{\left[A_{j k}\right]\left[m_{j}\right]=\left[c_{j}\right]}
\end{array}
$$




$$
\left[A_{j k}\right]\left[n_{j}\right]=\left[d_{j}\right]
$$

where $\left[A_{j k}\right]$ is the $4 \times 4$ coefficient matrix with $A_{j k}=\sum_{i=1}^{N} f_{k i} f_{j i}$ for $j, k=1,2,3$, 4, $\left[\ell_{j}\right]=\left[\begin{array}{llll}\ell_{1} & \ell_{2} & \ell_{3} & \ell_{4}\end{array}\right]^{T},\left[m_{j}\right]=\left[\begin{array}{llll}m_{1} & m_{2} & m_{3} & m_{4}\end{array}\right]^{T},\left[n_{j}\right]=\left[\begin{array}{llll}n_{1} & n_{2} & n_{3} & n_{4}\end{array}\right]^{T}$ and $\left[b_{j}\right]$, $\left[c_{j}\right]$ and $\left[d_{j}\right]$ are $4 \times 1$ matrices with $b_{j}=\sum_{i=1}^{N} F_{i} f_{j i}, c_{j}=-\sum_{i=1}^{N} f_{5 i} f_{j i}, d_{j}=-\sum_{i=1}^{N} f_{6 i} f_{j i}$ for $j=1,2,3,4$.

$\ell_{j}, m_{j}, n_{j}$ are uniquely solved from Eqs. (14-16). Once $\ell_{j}, m_{j}, n_{j}$ are determined, $\lambda_{1}$ and $\lambda_{2}$ are solved as follows:

$$
\begin{gathered}
\lambda_{1}=P_{3} P_{4}=\left(\ell_{3}+m_{3} \lambda_{1}+n_{3} \lambda_{2}\right)\left(\ell_{4}+m_{4} \lambda_{1}+n_{4} \lambda_{2}\right) \Rightarrow m_{3} m_{4} \lambda_{1}^{2}+n_{3} n_{4} \lambda_{2}^{2} \\
+\left(m_{3} n_{4}+m_{4} n_{3}\right) \lambda_{1} \lambda_{2}+\left(\ell_{3} m_{4}+\ell_{4} m_{3}-1\right) \lambda_{1}+\left(\ell_{3} n_{4}+\ell_{4} n_{3}\right) \lambda_{2}+\ell_{3} \ell_{4}=0 \\
\lambda_{2}=\frac{P_{5}}{P_{2}}=\frac{\lambda_{1}}{\left(\ell_{2}+m_{2} \lambda_{1}+n_{2} \lambda_{2}\right)} \Rightarrow n_{2} \lambda_{2}^{2}+m_{2} \lambda_{1} \lambda_{2}-\lambda_{1}+\ell_{2} \lambda_{2}=0
\end{gathered}
$$

We can solve for $\lambda_{1}$ from Eq. (18):

$$
\lambda_{1}=\frac{n_{2} \lambda_{2}^{2}+\ell_{2} \lambda_{2}}{\left(1-m_{2} \lambda_{2}\right)}
$$

Substituting Eq. (19) in Eq. (17):

$$
\begin{gathered}
m_{3} m_{4}\left(n_{2} \lambda_{2}^{2}+\ell_{2} \lambda_{2}\right)^{2}+n_{3} n_{4} \lambda_{2}^{2}\left(1-m_{2} \lambda_{2}\right)^{2}+\left(\ell_{3} n_{4}+\ell_{4} n_{3}\right) \lambda_{2}\left(1-m_{2} \lambda_{2}\right)^{2} \\
+\left(m_{3} n_{4}+m_{4} n_{3}\right)\left(n_{2} \lambda_{2}^{2}+\ell_{2} \lambda_{2}\right)\left(1-m_{2} \lambda_{2}\right) \lambda_{2}+\ell_{3} \ell_{4}\left(1-m_{2} \lambda_{2}\right)^{2} \\
+\left(\ell_{3} m_{4}+\ell_{4} m_{3}-1\right)\left(n_{2} \lambda_{2}^{2}+\ell_{2} \lambda_{2}\right)\left(1-m_{2} \lambda_{2}\right)=0
\end{gathered}
$$

Eq. (20) is a $4^{\text {th }}$ order polynomial equation in $\lambda_{2}$ and can be solved analytically. There may be 4,2 or no real solutions for $\lambda_{2}$. If exists, once one of the solutions for $\lambda_{2}$ is selected, $\lambda_{1}$ is determined uniquely from Eq. (19). $P_{j}=\ell_{j}+m_{j} \lambda_{1}+n_{j} \lambda_{2}$ for $j=1,2,3,4$ are determined and the construction parameters are solved uniquely from Eq. (3) as

$$
a=P_{2}, b=P_{3}, e=\frac{a}{P_{4}}, d=\sqrt{1+a^{2}+b^{2}+e^{2}+2 e P_{1}}
$$

\section{The Function Synthesis Problem}

Let the function to be generated be $z=f(x, y)$ for $x_{\text {min }} \leq x \leq x_{\max }$ and $y_{\min } \leq y$ $\leq y_{\max }$. The independent variables $x$ and $y$ should be related to the mechanism inputs $\theta$ and $\phi$ and the dependent variable $z$ should be related to the mechanism output $\psi . \theta, \phi$ and $\psi$ are in ranges $\theta_{\min } \leq \theta \leq \theta_{\max }, \phi_{\min } \leq \phi \leq \phi_{\max }$, $\psi_{\min } \leq \psi \leq \psi_{\max }$ and the limits can be arbitrarily chosen. We shall linearly relate $x$ to input $\theta, y$ to input $\phi$ and $z$ to output $\psi$ as [1] 


$$
\begin{aligned}
& \frac{x-x_{\min }}{x_{\max }-x_{\min }}=\frac{\theta-\theta_{\min }}{\theta_{\max }-\theta_{\min }} \\
& \frac{y-y_{\min }}{y_{\max }-y_{\min }}=\frac{\phi-\phi_{\min }}{\phi_{\max }-\phi_{\min }} \\
& \frac{z-z_{\min }}{z_{\max }-z_{\min }}=\frac{\psi-\psi_{\min }}{\psi_{\max }-\psi_{\min }}
\end{aligned}
$$

Then input values $\theta$ and $\phi$ and the desired output values $\psi$ of the mechanism can be solved from Eq. (21) as follows:

$$
\begin{aligned}
\theta & =\frac{x-x_{\text {min }}}{x_{\text {max }}-x_{\text {min }}}\left(\theta_{\text {max }}-\theta_{\text {min }}\right)+\theta_{\text {min }} \\
\phi & =\frac{y-y_{\text {min }}}{y_{\text {max }}-y_{\text {min }}}\left(\phi_{\text {max }}-\phi_{\text {min }}\right)+\phi_{\text {min }} \\
\psi & =\frac{z-z_{\text {min }}}{z_{\text {max }}-z_{\text {min }}}\left(\psi_{\text {max }}-\psi_{\text {min }}\right)+\psi_{\text {min }}
\end{aligned}
$$

and conversely

$$
\begin{aligned}
& x=\frac{\theta-\theta_{\text {min }}}{\theta_{\text {max }}-\theta_{\text {min }}}\left(x_{\text {max }}-x_{\text {min }}\right)+x_{\text {min }} \\
& y=\frac{\phi-\phi_{\text {min }}}{\phi_{\text {max }}-\phi_{\text {min }}}\left(y_{\text {max }}-y_{\text {min }}\right)+y_{\text {min }} \\
& z=\frac{\psi-\psi_{\text {min }}}{\psi_{\text {max }}-\psi_{\text {min }}}\left(z_{\text {max }}-z_{\text {min }}\right)+z_{\text {min }}
\end{aligned}
$$

We make use of Eq. (22) when determining the design points $\left\{\theta_{i}\right\}_{1}^{N},\left\{\phi_{i}\right\}_{1}^{N}$ and $\left\{\psi_{i}\right\}_{1}^{N}$ in terms of $\left\{x_{i}\right\}_{1}^{N},\left\{y_{i}\right\}_{1}^{N}$ and $\left\{z_{i}\right\}_{1}^{N}=\left\{f\left(x_{i}, y_{i}\right)\right\}_{1}^{N}$. We select the design points $\left(x_{i}, y_{i}\right)$ with equal spacing on the rectangular domain given by $x_{\min } \leq x \leq x_{\max }$ and $y_{\text {min }} \leq y \leq y_{\max }$, i.e. $x_{1}=x_{\text {min }}, y_{1}=y_{\min }, x_{i}=x_{\text {min }}+\frac{i-1}{N-1}\left(x_{\max }-x_{\text {min }}\right)$ and $y_{i}=y_{\min }+\frac{i-1}{N-1}\left(y_{\max }-y_{\text {min }}\right)$ for $i=2, \ldots, N$.

We make use of Eq. (23) after the synthesis is performed, to check the error in between the desired $z=f(x, y)$ and $z$ generated by the mechanism. At this step, one shall determine the output values of the mechanism for several given input values by solving the I/O relationship.

\section{Case Study}

The formulations in the previous sections were implemented in MS Excel ${ }^{\circledR}$ and a case study was worked out for a function $z=x^{1.1} y^{1.4}$ for $5 \leq x \leq 9$ and $1 \leq y \leq 4$. Limits for the mechanism input and output angles are selected as $75^{\circ} \geq \theta \geq 30^{\circ}$, $80^{\circ} \leq \phi \leq 130^{\circ}$ and $120^{\circ} \leq \psi \leq 170^{\circ}$. Actually several different limit values were employed, but the final selection is done until we obtain a small maximum error and 
good link length ratios. The design points are selected with equal spacing of 30 intervals for both $x$ and $y$. That is, there are totally 900 design points.

As a result of computations, there two real solutions for $\lambda_{2}$ are found. For one of the solutions the link lentgh ratios were not appropriate, whereas for the other solution a nice $5 \mathrm{R}$ mechanism is obtained. The maximum percentage error $100 \times\left|\frac{\psi_{\text {desired }}-\psi_{\text {computed }}}{\psi_{\text {desired }}}\right|$ is found as $1,33 \%$. The variation of the percentage error over the domain of $x$ and $y$ is illustrated in Figure 2. The construction parameters are calculated as $\mathrm{a}=2.382, \mathrm{~b}=1.636, \mathrm{~d}=2.671, \mathrm{e}=1.577$.

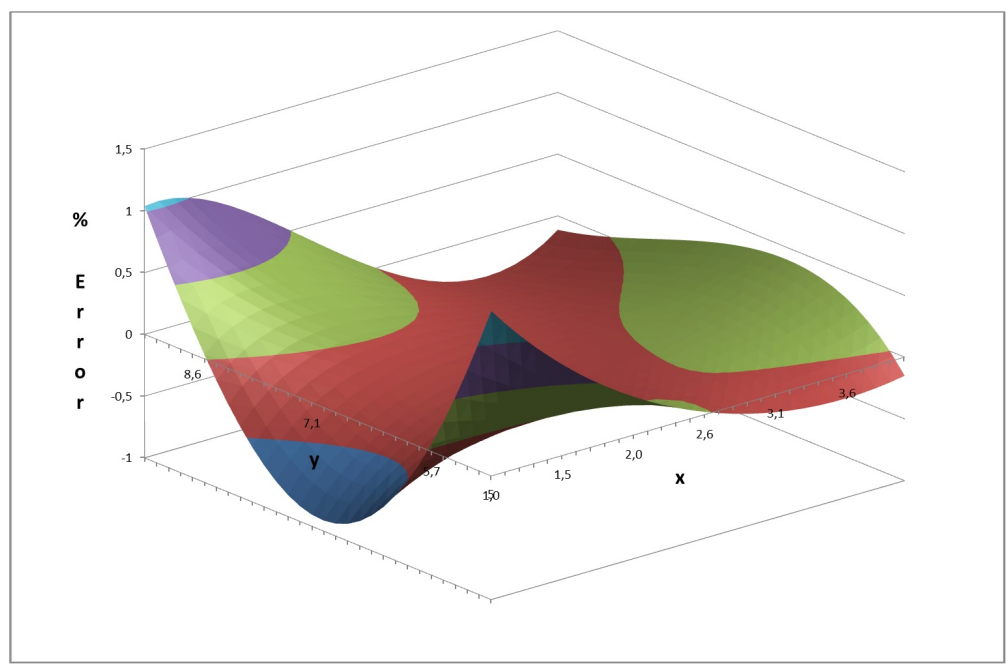

Fig. 2 The percentage error variation over the domain of $x$ and $y$

\section{Conclusions}

In this paper, the function generation problem for a planar $5 R$ mechanism is addressed using analytical solution for given set of design point. The problem is formulated starting with expressing the I/O relationship in polynomial form. The equations are linearized by introducing Lagrange variables. The linear set of equations and the Lagrange variables are solved analytically. A computational example is presented and the error variation is given.

As future studies we plan to work out the same problem using Chebyshev approximation and compare the results. Also we plan to apply these analytical and semi-analytical approximation methods to other multi-dof mechanisms. 


\section{References}

1. Erdman, A., Sandor, G. N.: Mechanism Design: Analysis and Synthesis, Prentice Hall (1984)

2. McCarthy, J. M., Soh, G. S.: Geometric Desgin of Linkages, $2^{\text {nd }}$ Ed., Springer (2011)

3. Alizade, R., Rao, A. V. M., Sandor, G. N.: Optimum synthesis of two-degree-of-freedom planar and spatial function generating mechanisms using the penalty function approach. Journal of Engineering for Industry 97(2), 629-634 (1975)

4. Svoboda, A.: Computing Mechanisms and Linkages, $2^{\text {nd }}$ Ed., Dover (1965)

5. Kim, H. S., Tsai, L.-W.: Kinematic synthesis of a spatial 3-RPS parallel manipulator. Journal of Mechanical Design 125, 92-97 (2003)

6. Kiper, G., Bilgincan, T., Dede, M.İ.C.: Function generation synthesis of planar 5R mechanism. Problems of Mechanics 2(51), 28-31 (2013)

7. Kiper, G., Bilgincan, T.: Function generation synthesis of spherical 5R mechanism. To appear in Mechanism and Machine Theory (2014)

8. Alizade, R., Gezgin, E.: Synthesis of function generating spherical four bar mechanism for the six independent parameters. Mechanism and Machine Theory 46, 1316-1326 (2011)

9. Levitskii, N.I., Sarkisian, E.Y.: On the special properties of Lagrange's multipliers in the leastsquare synthesis of mechanisms. Journal of Mechanisms 3(1), 3-10 (1968)

10. Alizade, R., Can, F.C., Kilit, Ö.: Least square approximate motion generation synthesis of spherical linkages by using Chebyshev and equal spacing. Mechanism and Machine Theory 61, 123-135 (2013)

11. Suixian, Y., Hong, Y., Tian, G.Y.: Optimal selection of precision points for function synthesis of spherical 4R linkage. Proceedings of the Institution of Mechanical Engineers, Part C: Journal of Mechanical Engineering Science 223, 2183-2189 (2009)

12. Alizade, R., Kiper, G., Bağdadioğlu, B., Dede, M.İ.C.: Function synthesis of Bennett 6R mechanisms using Chebyshev approximation. To appear in Mechanism and Machine Theory (2014) 